\title{
Dynamique de l'eau dans le rumen de vaches laitières: facteurs de variation et relation avec le transit des particules
}

\author{
M Mambrini, JL Peyraud \\ Station de Recherches sur la Vache Laitière, INRA, St-Gilles, 35590 L'Hermitage, France
}

Summary - Dilution rate and volume of water in the rumen varied between rations and dairy cows but were independent of the feed intake level. Correlations between rates of transit of liquid and particles were poor.

Introduction - Chez la vache laitière, peu d'études traitent de la dynamique de l'eau dans le rumen (Ørskov et al, 1988). Aussi avons nous étudié les variations du transit de l'eau dans le rumen et à l'entrée de l'intestin grêle ainsi que ses relations avec celui des particules.

Matériel et Méthodes - Les transits dans le rumen de l'eau et des particules, ainsi que le flux d'eau à l'entrée du duodénum ont été mesurés au cours de 3 essais (50 mesures) représentant au total 14 vaches Pie noires $(611 \mathrm{~kg})$ munies de canules du rumen et du duodénum. Les essais 1 et 2 ont été menés sur des vaches en milieu de lactation (respectivement 24 et 14 $\mathrm{kg}$ de lait 4\%). Dans l'essai 1, nous avons comparé 3 régimes (35 à $45 \%$ de concentré) à base d'ensilage de maïs, d'ensilage d'herbe et de foin, offerts à volonté et à $80 \%$ de ce niveau selon un schéma en inversion. Dans l'essai 2, nous avons étudié la digestion d'une herbe verte (ray grass anglais, cycle 2) d 2 stades (28 et $45 \mathrm{j}$ ), distribuée à volonté avec $2 \mathrm{~kg} / \mathrm{j}$ de concentré. Dans l'essai 3, l'effet du stade de lactation (sem $+4,32 \mathrm{~kg}$ lait $4 \%$; sem +20 , $21 \mathrm{~kg}$ lait 4\%; tarissement) a été étudié sur 4 vaches recevant à volonté la ration d'ensilage de maîs identique à celle de l'essai 1.

Le taux de renouvellement $\left(k_{\mathrm{liq}}\right.$ en $\left.\% / \mathrm{h}\right)$ et le volume ( $V$ en I) de l'eau du rumen, ainsi que le flux d'eau en sortie du rumen $\left(F I_{\text {r }}\right.$ en $\left.l / j\right)$ ont été estimés à partir de la décroissance de la concentration en polyéthylène-glycol (PEG) du liquide du rumen prélevé 2 et $26 \mathrm{~h}$ après une surcharge de 250 à $400 \mathrm{~g}$ de PEG. Les temps de séjour dans le rumen des particules de fourrage (marquées à l'ytterbium) et de concentré (marquées à l'europium) ont été estimés en ajustant les cinétiques d'excrétion fécale des marqueurs, distribués en dose unique au cours d'un repas test, selon la méthode de Grovum et Williams (1973). Le flux d'eau à l'entrée du duodénum $\left(F l_{d}\right.$ en $\left.l / j\right)$ a été estimé lors d'une infusion continue de PEG dans le rumen, à partir de la concentration moyenne en PEG de contenu de duodénum prélevé sur $3 \mathbf{j}$.

Résultats et Discussion - La variabilité des mesures de $V$ et de $F I_{r}$ ( $C V$ résiduelle $=22 \%$ ) a été supérieure à celle de $k_{\text {iq }}$ et de $F_{d}\left(C V_{r}: 10\right.$ et $14 \%$ respectivement). Les variations inter-individus ont été importantes pour $k_{\text {liq }}(1,3 \% / h), V$ (13 I) et $F l_{\mathrm{d}}(25 \mathrm{~V} / \mathrm{j})$. Le stade de lactation n'a pas modifié $k_{\text {liq }}$, mais $F_{\mathrm{r}}, F_{\mathrm{d}}$ et $V$ ont été plus faibles chez les animaux taris (tableau I). La limitation du niveau d'alimentation n'a pas eu d'effet sur $k_{\text {liq }}, V$ ou $F_{\text {r }}$, mais a entraîné une diminution de $15 \%$ de $F l_{\text {d. }}$. Avec l'herbe, $k_{\text {liq }}(16 \% / h)$ a été plus élevé qu'avec les régimes hivernaux $(12 \% / h)$ sans que $V$ ne soit modifié. Pour ces derniers, $k_{\text {liq }}$ du foin $(13 \% / h)$ a été supérieur à celui de l'ensilage d'herbe $(11 \% / h)$. 
Tableau I. Matière sèche (MSI) et eau consommées, taux de renouvellement $\left(k_{\text {liq }}\right)$, volume $(V)$, flux des liquides dans le rumen $\left(F I_{r}\right)$ et flux duodénal d'eau $\left(F I_{d}\right)$ mesurés sur des vaches laitières alimentées à volonté.

\begin{tabular}{|c|c|c|c|c|c|c|c|}
\hline Essai & $n$ & MSI (kg) & eau $(I / j)$ & $\mathrm{k}_{\text {liq }}(\% / h)$ & $V(l)$ & $\mathrm{FI}_{r}(1 / j)$ & $\mathrm{FI}_{d}(1 / j)$ \\
\hline $\begin{array}{l}1 \text { : foin } \\
\text { ens maïs } \\
\text { ens herbe }\end{array}$ & $\begin{array}{l}6 \\
4 \\
5\end{array}$ & $\begin{array}{l}18,0(14.6)^{\mathrm{a}} \\
18,4(15.4)^{\mathrm{a}} \\
16,7(14.5)^{\mathrm{b}}\end{array}$ & $\begin{array}{l}92(78) \\
90(79) \\
84(78)\end{array}$ & $\begin{array}{l}12,9^{\mathrm{a}} \\
12,5^{\mathrm{ab}} \\
10,9^{\mathrm{b}}\end{array}$ & $\begin{array}{l}70,0^{\mathrm{b}} \\
78,2^{\mathrm{ab}} \\
93,8^{\mathrm{c}}\end{array}$ & $\begin{array}{l}213 \\
230 \\
243\end{array}$ & $\begin{array}{l}279(231) \\
267(217) \\
255(236)\end{array}$ \\
\hline $\begin{array}{c}2: \text { herbe jeune } \\
\text { herbe âgée }\end{array}$ & $\begin{array}{l}4 \\
4\end{array}$ & $\begin{array}{l}14,2 \\
14,1\end{array}$ & $\begin{array}{l}92^{\mathrm{a}} \\
64^{\mathrm{b}}\end{array}$ & $\begin{array}{l}15,9 \\
17,3\end{array}$ & $\begin{array}{l}63,63 \\
69,63\end{array}$ & $\begin{array}{l}241^{a} \\
289^{b}\end{array}$ & $\begin{array}{l}295^{b} \\
255^{a}\end{array}$ \\
\hline $\begin{array}{l}3 \text { : début lact } \\
\text { milieu lact } \\
\text { tarissement }\end{array}$ & $\begin{array}{l}4 \\
4 \\
3\end{array}$ & $\begin{array}{l}19,3^{\mathrm{a}} \\
18,5^{\mathrm{a}} \\
13,8^{\mathrm{b}}\end{array}$ & $\begin{array}{l}88^{a} \\
89^{a} \\
41^{b}\end{array}$ & $\begin{array}{l}11,2 \\
10,8 \\
10,0\end{array}$ & $\begin{array}{l}78,7^{a} \\
97,5^{b} \\
57,7^{c}\end{array}$ & $\begin{array}{l}207^{a} \\
248^{a} \\
135^{b}\end{array}$ & $\begin{array}{l}253^{a} \\
255^{a} \\
206^{b}\end{array}$ \\
\hline $\begin{array}{l}\text { Ecart type individus* } \\
\text { Ecart type résiduel" }\end{array}$ & $\begin{array}{l}14 \\
14\end{array}$ & $\begin{array}{l}3,2 \\
2,3\end{array}$ & $\begin{array}{l}7 \\
8\end{array}$ & $\begin{array}{l}1,3 \\
1,2\end{array}$ & $\begin{array}{l}12,8 \\
16,1\end{array}$ & $\begin{array}{l}27 \\
46\end{array}$ & $\begin{array}{l}25 \\
31\end{array}$ \\
\hline
\end{tabular}

( ): Valeurs pour le niveau d'alimentation limite; a,b,c : significativement différents $(P<0.01)$ au sein de l'expérience. - De l'analyse de variance sur toutes les observations. $n$ : nombre d'animaux.

De tous les paramètres de la dynamique de l'eau étudiés, seuls $k_{\text {liq }}$ et $V$ sont corrélés $(r=-0,58)$. En moyenne, $F l_{d}$ est légèrement supérieur $( \pm 17 \mathrm{l} / \mathrm{j})$ mais non corrélé à $F I_{r}$, ni aux autres paramètres du rumen $\left(k_{\text {liq }}, \eta\right.$. $F l_{d}$ dépendrait donc plus des échanges d'eau à travers les parois du feuillet et de la caillette que des mouvements d'eau du rumen.

Les variations de $k_{\text {liq }}$ et de $V$ ne sont corrélées, ni à celles de la matière sèche ingérée, ni à celles de l'eau bue ou ingérée. Les seules variations d'ingestion n'expliquent donc pas celles du transit de l'eau, dépendant peut être davantage des mécanismes de régulation de la pression osmotique du contenu ruminal.

Le temps de séjour dans le rumen des particules de fourrages ou de concentré n'est que faiblement corrélé à $k_{\text {liq }}(r=-0,438$ et $-0,408$ respectivement). Le flux des petites particules n'est donc pas uniquement gouverné par celui de l'eau et dépendrait aussi des phénomènes de rétention dans les fibres plus longues des fourrages.

Grovum WL, Williams VJ (1973) Br J Nutr 30, 319-327

Ørskov ER, Ojwang I, Reid GW (1988) Anim Prod 47, 45-51 